\title{
Impurity-Bond Effect on the Spin-1 Antiferromagnetic Heisenberg Chain
}

\author{
Makoto Kaburagi, Isao Harada ${ }^{\dagger}$ and Takashi Tonegawa ${ }^{\dagger \dagger}$ \\ Department of Informatics, Faculty of Cross-Cultural Studies, \\ Kobe University, \\ Tsurukabuto, Nada, Kobe 657 \\ ${ }^{\dagger}$ Department of Physics, Faculty of Science, Okayama University, \\ Tsushima, Okayama 700 \\ ${ }^{\dagger \dagger}$ Department of Physics, Faculty of Science, Kobe University, \\ Rokkodai, Kobe 657
}

(Received December 24, 1992)

\begin{abstract}
Low-lying excited states of the spin-1 antiferromagnetic Heisenberg chain with an impurity bond are investigated in terms of domain-wall excitations by means of analytical methods as well as a method of numerical diagonalization. It is shown that 1) the impurity bond brings about a massive triplet mode in the Haldane gap, 2) the triplet state comprises three of the four ground states of an open chain, 3) the excited states with the domain wall trapped at the impurity bond, including the massive triplet mode, exhibit a Néel-type configuration of the magnetic moment around the impurity bond. On the basis of the present results, we propose a new energy versus magnetic-field diagram.
\end{abstract}


The spin-1 antiferromagnetic Heisenberg chain has been the subject of a large number of theoretical and experimental studies since Haldane's prediction $^{1)}$ of the difference between integer-spin and half-integer-spin antiferromagnetic Heisenberg chains. Most of the theoretical studies done so far have been concerned with the properties of the periodic chain. Recently Kennedy $^{2}$ found that the open chain has a fourfold degenerate ground state composed of a singlet and a triplet which we call the Kennedy triplet, in contrast to a unique singlet ground state of the periodic chain. ${ }^{3)}$ The fourfold degeneracy of the ground state, which was originally found in the so-called AKLT model ${ }^{3)}$ with open boundary conditions, is considered to reflect the hidden $Z_{2} \times Z_{2}$ symmetry in the open chain. ${ }^{4)}$

In this letter we investigate low-lying excited states which include the Kennedy triplet of the spin-1 antiferromagnetic Heisenberg chain with an impurity bond. The impurity bond plays a mediating role between the open chain and the periodic chain. Using analytical methods as well as a method of numerical diagonalization, we calculate the energies of the low-lying excited states and also the magnetic moment at each site of the Kennedy triplet. In particular, exploring the dependence on the impuritybond strength of the energy difference between the Kennedy triplet and the singlet is interesting and important, since it yields information on the transition of the ground state from the fourfold to the unique one.

Before going into the details of the calculation, we briefly discuss our physical picture of the impurity-bond effect. It is convenient in many cases to express elementary excitations in the antiferromagnetic Heisenberg chain in terms of domain-wall excitations. If the excited state of the spin-1 periodic chain is the sliding mode of domain-wall excitations, it is expected, analogously to the spin-1/2 Ising-like periodic chain, ${ }^{5)}$ that the impurity 
bond traps the domain wall and brings about the massive triplet mode below the bottom of the energy continuum. For the open chain, this massive mode should comprise three of the four ground states. It is also expected that the excited states with the trapped domain wall, including the massive triplet mode, exhibit a Néel-type configuration of the magnetic moment around the impurity bond.

We express the Hamiltonian which describes the spin-1 antiferromagnetic Heisenberg chain with an impurity bond as

$$
\begin{aligned}
& \mathcal{H}=\sum_{\ell=1}^{N-1} h_{\ell, \ell+1}+\kappa h_{N, 1}+D \sum_{\ell=1}^{N}\left(S_{\ell}^{z}\right)^{2}, \\
& h_{\ell, \ell^{\prime}}=2\left(S_{\ell}^{x} S_{\ell^{\prime}}^{x}+S_{\ell}^{y} S_{\ell^{\prime}}^{y}+\lambda S_{\ell}^{z} S_{\ell^{\prime}}^{z}\right)+2 K\left(\vec{S}_{\ell} \cdot \vec{S}_{\ell^{\prime}}\right)^{2} \quad(\lambda>0),
\end{aligned}
$$

where $\kappa$ represents the strength of the impurity bond, with $\kappa=0$ and $\kappa=1$ corresponding, respectively, to the open chain and the periodic chain. For convenience, we denote as $E_{\mathrm{s}}$ the energy of the state corresponding to the singlet ground state at $\lambda=\kappa=1$ and $D=K=0$, and also as $E_{\mathrm{t}}^{(\tau)}$ the energies of the states corresponding to the Kennedy triplet at $\lambda=1$ and $\kappa=D=K=0$, where $\tau=0$ for the $S_{\text {total }}^{z} \equiv \sum_{\ell=1}^{N} S_{\ell}^{z}=0$ state and $\tau= \pm$ for the $S_{\text {total }}^{z}= \pm 1$ states. Furthermore, we set $\Delta_{0}(\kappa) \equiv E_{\mathrm{t}}^{(0)}-E_{\mathrm{s}}$ and $\Delta_{1}(\kappa) \equiv E_{\mathrm{t}}^{( \pm)}-E_{\mathrm{s}}$.

We first calculate, by means of a variational method, $E_{\mathrm{s}}$ and $\Delta_{i}(\kappa)$ $(i=0,1)$ for the case of $|\kappa| \ll 1$. We introduce the following matrix-productform wave functions $\Phi, \Phi_{n}^{(+)}, \Phi_{n}^{(0)}$, and $\Phi_{n}^{(-)}$, generalizing that of Klümper et $a l .{ }^{6)}$ which was originally proposed by Fannes et al. $\left.{ }^{7}\right)$ The function $\Phi$ describes the state with no domain wall and is defined as

$$
\begin{aligned}
\Phi= & \operatorname{Trace}\left[\phi_{1} \phi_{2} \cdots \phi_{N-1} \phi_{N}\right], \\
& \phi_{\ell}=\cos \left(\theta_{\ell}\right) \zeta_{\ell} \sigma_{z}+\frac{\sin \left(\theta_{\ell}\right)}{\sqrt{2}}\left(\alpha_{\ell} \sigma_{+}+\beta_{\ell} \sigma_{-}\right),
\end{aligned}
$$


where $\alpha_{\ell}, \zeta_{\ell}$, and $\beta_{\ell}$ are the spin states at the $\ell$-th site, which correspond, respectively, to $S_{\ell}^{z}=1,0$, and -1 , and $\sigma_{ \pm}\left[=\left(\sigma_{x} \pm i \sigma_{y}\right) / \sqrt{2}\right], \sigma_{x}, \sigma_{y}$, and $\sigma_{z}$ are the Pauli matrices. On the other hand, the function $\Phi_{n}^{(\tau)}$ describes the states with a domain wall at the $n$-th bond between the $n$th and $(n+1)$ th sites, and is defined in terms of $\phi_{\ell}$ and an operator $w$, which may be called the wall operator, as

$$
\Phi_{n}^{(\tau)}=\operatorname{Trace}\left[\phi_{1} \phi_{2} \cdots \phi_{n} w \phi_{n+1} \cdots \phi_{N-1} \phi_{N}\right]
$$

where $w=-\sigma_{-}$for $\tau=+, w=\sigma_{z}$ for $\tau=0$, and $w=\sigma_{+}$for $\tau=-$. It is noted that when $\lambda=1, \Phi$ represents the singlet state, and $\Phi_{n}^{(+)}, \Phi_{n}^{(0)}$, and $\Phi_{n}^{(-)}$represent, respectively, the triplet states with $S_{\text {total }}^{z}=1,0$, and -1 . In the case of $|\kappa| \ll 1$ the domain wall is expected to be trapped at the impurity bond, i.e., the $N$ th bond. We therefore use $\Phi, \Phi_{N}^{(+)}, \Phi_{N}^{(0)}$, and $\Phi_{N}^{(-)}$as trial functions. For simplicity, we assume that $\theta_{\ell}=\theta$ for all $\ell$ 's. Then, these trial functions become equivalent to that used by Kennedy and Tasaki. ${ }^{4}$ ) We have carried out a variational calculation with respect to $\theta$ for the energy expectation values. Up to the order of $N$, the four trial functions lead to the same solution $\theta=\tilde{\theta}$, where $\tilde{\theta}$ is given by

$$
\tilde{\theta}= \begin{cases}0 & \text { for } \quad D \geq \operatorname{Max}(4, \lambda) \\ \frac{1}{2} \arccos \left(\frac{D-\lambda}{4-\lambda}\right) & \text { for } 4>D>2 \lambda-4 \\ \frac{\pi}{2} & \text { for } \quad \operatorname{Min}(\lambda, 2 \lambda-4) \geq D\end{cases}
$$

This solution in the region $D \geq \operatorname{Max}(4, \lambda)$, that in the region $4>D>2 \lambda-4$, and that in the region $\operatorname{Min}(\lambda, 2 \lambda-4) \geq D$ correspond, respectively, to the large- $D$, Haldane, and Néel phases. (In the calculation for the Haldane phase we have neglected the factors $\cos ^{N} \theta$ and $\cos ^{N} 2 \theta$.) We denote $\Phi$ and $\Phi_{N}^{(\tau)}$ for $\theta=\tilde{\theta}$ by $\tilde{\Phi}$ and $\tilde{\Phi}_{N}^{(\tau)}$, respectively. It is noted that $\tilde{\Phi}$ and $\tilde{\Phi}_{N}^{(\tau)}$ for 
$D=0$ and $\lambda=1$ are equivalent to the valence-bond-solid ${ }^{3)}$ wave functions expressed by the Schwinger bosons. ${ }^{8)}$

Let us concentrate our attention on the Haldane phase. For this phase, the variational results for $E_{\mathrm{s}}$ and $\Delta_{i}(\kappa)$ are given by

$$
\begin{aligned}
E_{\mathrm{s}} & \simeq\langle\tilde{\Phi}|\mathcal{H}| \tilde{\Phi}\rangle \\
& =-(N-1+\kappa)\left\{\left(\frac{4-D}{4-\lambda}\right)\left(1+\frac{D}{4}\right)+2 K\right\}+N \frac{D(4-D)}{2(4-\lambda)}, \\
\Delta_{0}(\kappa) & \simeq\left\langle\tilde{\Phi}_{N}^{(0)}|\mathcal{H}| \tilde{\Phi}_{N}^{(0)}\right\rangle-E_{\mathrm{s}} \\
& =\kappa\left\{\frac{2(4-D)(4-2 \lambda+D)}{(4-\lambda)^{2}}-K\right\} \equiv \kappa \tilde{\Delta}_{0}, \\
\Delta_{1}(\kappa) & \simeq\left\langle\tilde{\Phi}_{N}^{( \pm)}|\mathcal{H}| \tilde{\Phi}_{N}^{( \pm)}\right\rangle-E_{\mathrm{s}} \\
& =\kappa \frac{4-D}{4(4-\lambda)^{2}}\{2(8+2 D-\lambda D)-K(12-4 \lambda+D)\} \equiv \kappa \tilde{\Delta}_{1} .
\end{aligned}
$$

When $\kappa=0$, as is expected, the four states $\tilde{\Phi}$ and $\tilde{\Phi}_{N}^{(\tau)}$ are degenerate in energy, which reflects the hidden $Z_{2} \times Z_{2}$ symmetry in the open chain. This degeneracy is resolved linearly with respect to $\kappa$; in the case of $\kappa>0$, either $0<\Delta_{1}(\kappa)<\Delta_{0}(\kappa)$ or $0<\Delta_{0}(\kappa)<\Delta_{1}(\kappa)$ depending on whether $D(4+2 \lambda-3 K)$ is larger or smaller than $4(4-K)(\lambda-1)$, and in the case of $\kappa<0$, either $0>\Delta_{1}(\kappa)>\Delta_{0}(\kappa)$ or $0>\Delta_{0}(\kappa)>\Delta_{1}(\kappa)$ depending on whether $D(4+2 \lambda-3 K)$ is larger or smaller than $4(4-K)(\lambda-1)$. Thus, when $\lambda=1$ and $D=0$, a transition of the ground state from a singlet to a triplet takes place at $\kappa=0$ with increasing $\kappa$. This finding indicates that the impurity bond plays the role of the $Z_{2} \times Z_{2}$ symmetry breaking field and that the $Z_{2} \times Z_{2}$ symmetry is not a characteristic property of the bulk.

The site dependence of the magnetic moments is calculated as

$$
\begin{aligned}
& \left\langle\tilde{\Phi}\left|S_{\ell}^{z}\right| \tilde{\Phi}\right\rangle=\left\langle\tilde{\Phi}_{N}^{(0)}\left|S_{\ell}^{z}\right| \tilde{\Phi}_{N}^{(0)}\right\rangle=0 \\
& \left\langle\tilde{\Phi}_{N}^{(+)}\left|S_{\ell}^{z}\right| \tilde{\Phi}_{N}^{(+)}\right\rangle=-\left\langle\tilde{\Phi}_{N}^{(-)}\left|S_{\ell}^{z}\right| \tilde{\Phi}_{N}^{(-)}\right\rangle=\sin ^{2} \tilde{\theta}\left(\cos ^{\ell-1} 2 \tilde{\theta}+\cos ^{N-\ell} 2 \tilde{\theta}\right) .
\end{aligned}
$$


The result given by eq. (10), which is essentially the same as that obtained by Kennedy and Tasaki, ${ }^{4)}$ indicates that $\left\langle\tilde{\Phi}_{N}^{( \pm)}\left|S_{\ell}^{z}\right| \tilde{\Phi}_{N}^{( \pm)}\right\rangle$decays exponentially with the decay constant $-(\ln |\cos 2 \tilde{\theta}|)^{-1}$ as a function of the distance from the impurity bond.

We briefly discuss the case in which the magnetic-field term $\mathcal{H}_{\mathrm{mf}}=$ $-H_{x} S_{\text {total }}^{x}-H_{z} S_{\text {total }}^{z}\left(S_{\text {total }}^{x} \equiv \sum_{\ell=1}^{N} S_{\ell}^{x}\right)$ is added to the Hamiltonian $\mathcal{H}$. The energy difference $\Delta E$ (the energy measured from $E_{\mathrm{s}}$ ) versus magnetic field $H_{x}$ or $H_{z}$ diagram can be constructed by solving the equation,

$$
\left[\Delta_{0}(\kappa)-\Delta E\right]\left[\left\{\Delta_{1}(\kappa)-\Delta E\right\}^{2}-H_{z}^{2}\right]-G^{2} H_{x}^{2}\left\{\Delta_{1}(\kappa)-\Delta E\right\}=0
$$

with

$$
G=\left\langle\tilde{\Phi}_{N}^{(+)}\left|S_{\text {total }}^{x}\right| \tilde{\Phi}_{N}^{(0)}\right\rangle=\left\langle\tilde{\Phi}_{N}^{(-)}\left|S_{\text {total }}^{x}\right| \tilde{\Phi}_{N}^{(0)}\right\rangle=\sin 2 \tilde{\theta} /\left(1+\cos ^{2} 2 \tilde{\theta}\right)
$$

We note here that $\left\langle\tilde{\Phi}\left|S_{\text {total }}^{x}\right| \tilde{\Phi}_{N}^{(+)}\right\rangle=\left\langle\tilde{\Phi}\left|S_{\text {total }}^{x}\right| \tilde{\Phi}_{N}^{(0)}\right\rangle=\left\langle\tilde{\Phi}\left|S_{\text {total }}^{x}\right| \tilde{\Phi}_{N}^{(-)}\right\rangle=$ $\left\langle\tilde{\Phi}_{N}^{(+)}\left|S_{\text {total }}^{x}\right| \tilde{\Phi}_{N}^{(-)}\right\rangle=0$. The explicit results for the solution of eq. (11) will be discussed in a separate paper. ${ }^{9)}$

Next we deal with the case of $\kappa \lesssim 1$, again concentrating our attention on the Haldane phase. In this case it is important to take into account the effect of the propagation of domain walls in the chain. When $\kappa \neq 1$, the scattering of propagating domain walls by the impurity bond produces a domain-wall bound state around it. To discuss this bound state, we employ Koster and Slater's method. ${ }^{10)}$ (A more sophisticated treatment will be discussed elsewhere. ${ }^{11)}$ ) This method leads to the following result for the energy differences $\Delta_{i}(\kappa)$ :

$$
\Delta_{i}(\kappa) \simeq \Delta_{i}(1)-a_{i}(1-\kappa)^{2},
$$


where $\Delta_{i}(1)$ and $a_{i}$ are constants, the latter being a quantity related to the matrix elements of $h_{N, 1}$, and are determined phenomenologically below. The present Koster-Slater analysis also leads to the result that the energy difference between the bottom of the energy continuum for $S_{\text {total }}^{z}=0$ and the ground state and that between the bottom of the energy continuum for $S_{\text {total }}^{z}= \pm 1$ and the ground state are given, respectively, by $\Delta_{0}(1)$ and $\Delta_{1}(1)$. Accordingly, these energy differences are independent of $\kappa$.

We assume that eq. (13) also holds for $\kappa \sim 0$ to determine $\Delta_{i}(1)$ and $a_{i}$. Then, $a_{i}$ should be equal to $\Delta_{i}(1)$, since $\Delta_{i}(\kappa)$ should vanish at $\kappa=0$. Thus, we have

$$
\Delta_{i}(\kappa)=\Delta_{i}(1)\left\{1-(1-\kappa)^{2}\right\}
$$

Furthermore, if we use the variational results given by eqs. (7) and (8), $\Delta_{i}(1)$ is determined to be $\Delta_{i}(1)=\tilde{\Delta}_{i} / 2$.

On the basis of the results of the above Koster-Slater analysis, we discuss how the state at the bottom of the energy continuum changes with $\kappa$, considering, for simplicity, the isotropic case of $\lambda=1$ and $D=K=0$ in which $\Delta_{0}(\kappa)=\Delta_{1}(\kappa) \equiv \Delta(\kappa)$. At $\kappa=1$ the energy difference between the bottom of the continuum for the triplet state and that for the quintet state is considered to be equal to $\Delta(1)$. On the other hand, the binding energy of one trapped domain wall at $\kappa=0$ is given by $\Delta(1)$ [see eq. (14)], and the energy difference $\Delta(1)$ between the bottom of the continuum for the triplet state and the ground state is independent of $\kappa$. Thus, when the value of $\kappa$ decreases, the state at the bottom of the continuum is expected to change at $\kappa=0$ from the triplet to the quintet, since at $\kappa \sim 0$ the latter state is constructed by adding one trapped domain wall to the former state.

Combining these considerations with the variational result that $\Delta(\kappa)$ 
is proportional to $\kappa$ at $\kappa \sim 0$ [see eqs. (7) and (8)] and also with the result given by eq. (14), we conjecture that the energy (measured from $E_{\mathrm{s}}$ ) versus $\kappa$ diagram is that which is schematically given in Fig. 1 . Here we have assumed that the energy difference between the bottom of the continuum and the ground state remains unchanged also for $\kappa<0$. According to this diagram the Haldane gap is defined as the energy difference between the bottom of the energy continuum and the ground state for any $\kappa$.

In order to numerically examine the analytical results as well as the conjecture discussed above, we have performed diagonalizations by Lanczös method for finite- $N$ chains in the isotropic case. In Fig. 2 we plot $\Delta(\kappa) / \Delta(1)$ as a function of $\kappa^{\prime}\left[\equiv\left(\kappa-\kappa_{\mathrm{c}}\right) /\left(1-\kappa_{\mathrm{c}}\right)\right]$ for $N=12$ and $N=13$, where $\kappa_{\mathrm{c}}$ is the value of $\kappa$ at which $\Delta(\kappa)$ vanishes for each $N$. The corresponding plot of eq. (14), where $\kappa^{\prime}=\kappa$, is also given in Fig. 2. From this figure we see that the result for the $\kappa$ dependence of $\Delta(\kappa)$ obtained by the above single domain-wall analysis is in rather good agreement with the numerical results. In Fig. 3 the energies (measured from $E_{\mathrm{s}}$ ) of low-lying excited states for $N=13$ are plotted as a function of $\kappa$; the dotted line is for the lowest triplet state [i.e., the dotted line shows $\Delta(\kappa)$ ], the dashed line is for the second-lowest triplet state, and the dot-dashed line is for the lowest quintet state. This figure should be compared with Fig. 1. We plot in Fig. 4 the $\ell$-dependence of the magnetic moments $\left\langle S_{\ell}^{z}\right\rangle$ of the $S_{\text {total }}^{z}=1$ state for the lowest triplet and of the $S_{\text {total }}^{z}=2$ state for the lowest quintet obtained for $N=13$ and $\kappa=0$ (the open chain), where $\langle\cdots\rangle$ stands for the expectation value with respect to a given state. This figure suggests a Néeltype configuration of the magnetic moments around the impurity bond for both states. It should be noted that the magnetic moments shown in Fig. 4 are quite different from $\left\langle S_{\ell}^{z}\right\rangle=S_{\text {total }}^{z} / N$ for $\kappa=1$ (the periodic chain) and 
that the values of the magnetic moments at the edges of the open chain are about 60 percent of the value 1 of the full moment. As may be seen from Fig. 4, the decay constant of the $S_{\text {total }}^{z}=1$ state for the lowest triplet is much longer than the variational result of $-(\ln |\cos 2 \tilde{\theta}|)^{-1} \sim 0.9$. In fact, Miyashita and Yamamoto $^{12)}$ have very recently shown that the $N \rightarrow \infty$ value of the decay constant of this state is given by about 6 . Details of the numerical calculation will be published in the near future. ${ }^{11)}$

In conclusion, we emphasize that the Haldane gap should not be defined as the singlet-triplet excitation energy, but as the energy difference between the bottom of the energy continuum and the ground state. We propose, for the isotropic open chain, the energy (measured from $E_{\mathrm{s}}$ ) versus magneticfield $H_{z}$ diagram given by Fig. 5 (see Fig. 1 also). It is noted that the critical field $H_{z}^{\mathrm{c}}$, at which the finite magnetization per site appears, is equal to $\Delta(1)$ as in the case of the isotropic periodic chain. Furthermore, we expect that for samples doped by nonmagnetic impurities of a few or more percent, the magnetic response due to the Néel-type configuration of magnetic moment near the edges can be observed at a magnetic field even below $H_{z}^{c}$. This may be an important subject for future experimental studies for examining our physical picture.

The present work has been supported in part by a Grant-in-Aid for Scientific Research on Priority Areas, "Computational Physics as a New Frontier in Condensed Matter Research", from the Ministry of Education, Science and Culture. One of the authors (M. K.) gratefully acknowledges the support of Fujitsu Limited. 


\section{References}

1) F. D. M. Haldane: Phys. Lett. 93A (1983) 464; Phys. Rev. Lett. 50 (1983) 1153.

2) T. Kennedy: J. Phys. Condens. Matter 2 (1990) 5737.

3) I. Affleck, T. Kennedy, E. H. Lieb and H. Tasaki: Phys. Rev. Lett. 59 (1987) 799; Commun. Math. Phys. 115 (1988) 477.

4) T. Kennedy and H. Tasaki: Commun. Math. Phys. 147 (1992) 431.

5) T. Moriwaki, M. Kaburagi and T. Tonegawa: unpublished.

6) A. Klümper, A. Schadschneider and J. Zittartz: J. Phys. A 24 (1991) L955; Z. Phys. B 87 (1992) 281.

7) M. Fannes, B. Nachtergaele and R. F. Werner: Europhys. Lett. 10 (1989) 633; Commun. Math. Phys. 144 (1992) 443.

8) D. P. Arovas and A. Auerbach: Phys. Rev. B 38 (1988) 316.

9) M. Kaburagi and T. Tonegawa: in preparation.

10) G. F. Koster and J. C. Slater: Phys. Rev. 96 (1954) 1208.

11) M. Kaburagi: in preparation.

12) S. Miyashita and S. Yamamoto: preprint. 


\section{Figure Captions}

Fig. 1. Schematic energy (measured from $E_{\mathrm{s}}$ ) versus $\kappa$ diagram for the isotropic case obtained by analytical methods. The dotted line is for the lowest triplet state and the dashed (dot-dashed) line is for the bottom of the energy continuum consisting of triplet (quintet) states. The gray region shows the energy continuum.

Fig. 2. Plots of $\Delta(\kappa) / \Delta(1)$ as a function of $\kappa^{\prime}\left[\equiv\left(\kappa-\kappa_{\mathrm{c}}\right) /\left(1-\kappa_{\mathrm{c}}\right)\right]$ for $N=12$ (open circles) and $N=13$ (open squares) in the isotropic case, where $\kappa_{\mathrm{c}}$ is the value of $\kappa$ at which $\Delta(\kappa)$ vanishes for each $N$. The solid line is the corresponding plot of eq. (14), where $\kappa^{\prime}=\kappa$.

Fig. 3. Energy (measured from $E_{\mathrm{s}}$ ) versus $\kappa$ diagram for $N=13$ in the isotropic case obtained by numerical diagonalization. The dotted line is for the lowest triplet state, the dashed line is for the second-lowest triplet state, and the dot-dashed line is for the lowest quintet state.

Fig. 4. Plots versus $\ell$ of the magnetic moments $\left\langle S_{\ell}^{z}\right\rangle$ of the $S_{\text {total }}^{z}=1$ state for the lowest triplet (full circles) and of the $S_{\text {total }}^{z}=2$ state for the lowest quintet (open circles) obtained for $N=13$ and $\kappa=0$.

Fig. 5. Energy (measured from $E_{\mathrm{s}}$ ) versus magnetic-field $H_{z}$ diagram proposed for the isotropic open chain. 